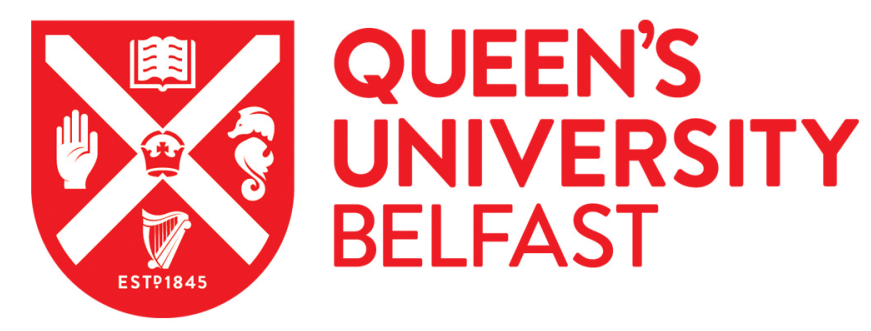

\title{
Hollow Gold Nanoparticles Produced by Femtosecond Laser Irradiation
}

Castro-Palacio, J. C., Ladutenko, K., Prada, A., González-Rubio, G., Díaz-Núñez, P., Guerrero-Martínez, A., Fernández de Córdoba, P., Kohanoff, J. J., Perlado, J. M., Peña-Rodríguez, O., \& Rivera, A. (2020). Hollow Gold Nanoparticles Produced by Femtosecond Laser Irradiation. Journal of Physical Chemistry Letters, 11(13), 5108-5114. https://doi.org/10.1021/acs.jpclett.0c01233

Published in:

Journal of Physical Chemistry Letters

Document Version:

Peer reviewed version

Queen's University Belfast - Research Portal:

Link to publication record in Queen's University Belfast Research Portal

Publisher rights

Copyright $2020 \mathrm{ACS}$. This work is made available online in accordance with the publisher's policies. Please refer to any applicable terms of use of the publisher.

\section{General rights}

Copyright for the publications made accessible via the Queen's University Belfast Research Portal is retained by the author(s) and / or other copyright owners and it is a condition of accessing these publications that users recognise and abide by the legal requirements associated with these rights.

Take down policy

The Research Portal is Queen's institutional repository that provides access to Queen's research output. Every effort has been made to ensure that content in the Research Portal does not infringe any person's rights, or applicable UK laws. If you discover content in the Research Portal that you believe breaches copyright or violates any law, please contact openaccess@qub.ac.uk. 


\title{
Hollow gold nanoparticles produced by femtosecond
}

\section{laser irradiation}

\author{
Juan Carlos Castro-Palacio, ${ }^{\infty, *}$ Konstantin Ladutenko, ${ }^{\S}$ Alejandro Prada,,$^{\#, \dagger}$ \\ Guillermo González-Rubio, ${ }^{+}$Pablo Díaz-Núñez, ${ }^{\star A n d r e ́ s ~ G u e r r e r o-M a r t i ́ n e z, ~}{ }^{,+}$ \\ Pedro Fernández de Córdoba, ${ }^{*}$ Jorge Kohanoff, ${ }^{\top}$ José Manuel Perlado,,${ }^{\natural}$ \\ Ovidio Peña-Rodríguez ${ }^{\mathrm{\alpha}, \diamond, *}$ and Antonio Rivera ${ }^{\mathrm{\alpha}, \diamond, *}$
}

"Instituto de Fusión Nuclear “Guillermo Velarde”, Universidad Politécnica de Madrid, José Gutiérrez Abascal 2, E-28006 Madrid, Spain

${ }^{*}$ Grupo de Modelización Interdisciplinar, InterTech, Instituto Universitario de Matemática Pura y Aplicada, Universitat Politècnica de València, Camino de Vera, s/n, 46022 València, Spain

${ }^{\S}$ Department of Physics and Engineering, ITMO University, 49 Kronverskii Ave., St. Petersburg 197101, Russian Federation

\#Departamento de Computación e Ingenierías, Facultad de Ciencias de la Ingeniería, Universidad Católica del Maule, Talca, Chile

${ }^{\dagger}$ Centro de Nanotecnología Aplicada, Facultad de Ciencias, Universidad Mayor, Santiago, Chile

\$Departamento de Química Física, Universidad Complutense de Madrid, Avenida Complutense s/n, 28040 Madrid, Spain 
₹ASC, School of Mathematics and Physics, Queen's University Belfast, Belfast BT7 1NN, Northern Ireland, United Kingdom

${ }^{\ominus}$ Departamento de Ingeniería Energética, ETSII Industriales, Universidad Politécnica de Madrid, José Gutiérrez Abascal 2, E-28006 Madrid, Spain

\section{AUTHOR INFORMATION}

\section{Corresponding Authors}

*Email: antonio.rivera@upm.es (A. R.), ovidio.pena@upm.es (O. P.-R.) 


\section{ABSTRACT}

Metallic hollow nanoparticles exhibit interesting optical properties that can be controlled by geometrical parameters. Irradiation with femtosecond laser pulses has emerged recently as a valuable tool for reshaping and size modification of plasmonic metal nanoparticles, thereby enabling the synthesis of nanostructures with unique morphologies. In this letter, we use classical molecular dynamics simulations to investigate the solid-to-hollow conversion of gold nanoparticles upon femtosecond laser irradiation. Here, we suggest an efficient method to produce hollow nanoparticles under certain specific conditions, namely that the particles should be heated to a maximum temperature between 2500 and $3500 \mathrm{~K}$, followed by a fast quenching to room temperature, with cooling rates below 120 ps. Therefore, we advance the experimental conditions to efficiently produce hollow nanoparticles, opening a broad range of possibilities for applications in key areas, such as energy storage and catalysis.

\section{TOC GRAPHICS}

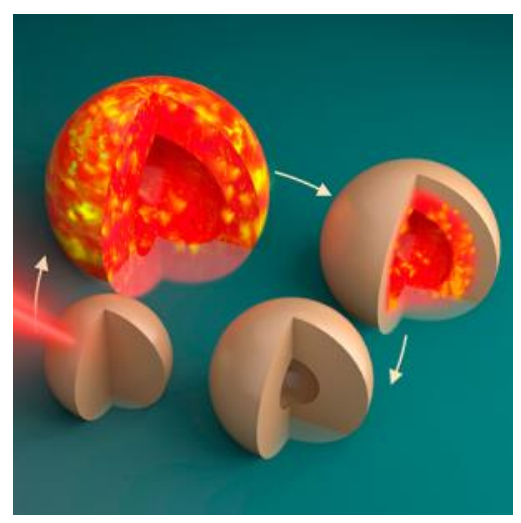

KEYWORDS. hollow nanoparticles, localized surface plasmon resonance, femtosecond laser pulses. 
The collective oscillation of electrons in the conduction band of metal nanoparticles, a phenomenon known as localized surface plasmon resonance (LSPR), has drawn the attention of many research groups over the past decades. There have been numerous works on the fabrication ${ }^{1-}$ ${ }^{4}$ and characterization ${ }^{5-8}$ of plasmonic nanostructures aimed at the modification of their optical properties to develop new potential applications. ${ }^{9-11}$ Among those applications, we can mention catalysis, ${ }^{12,13}$ biological and chemical sensing, ${ }^{14,15}$ photonics and energy harvesting, ${ }^{16-20}$ and storage. ${ }^{21}$ Gold and silver have been intensively studied, owing to their ability of sustaining narrow and intense plasmonic resonances. ${ }^{22}$ Besides noble metal nanoparticles and their alloys, ${ }^{23}$ the list of previously studied nanostructures includes copper chalcogenide nanocrystals, ${ }^{24-27}$ metal oxides ${ }^{28}$ and transition metal oxides. ${ }^{29}$

Another relevant topic is the study of the effects related to the high electronic excitation, produced when plasmonic nanoparticles are irradiated with ultrashort laser pulses or swift heavy ions. ${ }^{40-32}$ This approach has been used to fabricate metal nanostructures covering a broad spectrum of applications, such as aligned nanorods, ${ }^{31}$ nanorod dimers,${ }^{30}$ improved nanorod colloids, ${ }^{4,33}$ and hollow nanoparticles ${ }^{34}$. Among the applications, hydrogen storage and release is particularly interesting in the current context of global warming. ${ }^{35,36}$ The possibility of controlling the shape as a function of laser fluence, pulse duration, and nanoparticle geometry are key aspects of the design and fabrication of nanoparticles with tailored plasmonic properties. For example, we used femtosecond pulses to reshape gold nanorods in different ways. ${ }^{4,33}$ Likewise, we showed recently that irradiation with nanosecond laser pulses can be used to produce hollow gold nanospheres. ${ }^{34}$

In this article, we carry out molecular dynamics (MD) simulations to investigate the shape dynamics of gold nanoparticles after single-shot irradiation with ultrashort laser pulses. We also 
discuss the irradiation and energy dissipation conditions (dictated by the characteristics of the environment) required to form these hollow particles. Our simulations suggest that it is possible to induce the formation of cavities inside the irradiated particles solely with the irradiation, (i.e., with no need of stabilization by uptake of external matter, as demanded with nanosecond pulse irradiation). The conditions required here differ considerably from those in our previous paper ${ }^{34}$ because, from the point of view of the energy transfer from the electronic system to the lattice, femtosecond and nanosecond pulses represent two extreme cases. ${ }^{37}$ Pulses with a duration around $100 \mathrm{fs}$, typical for Ti:sapphire lasers, are much shorter than the time constant of electron-phonon coupling $\left(\tau_{e-p}\right)$. Hence, the energy is deposited almost adiabatically on the electronic system. On the other hand, nanosecond pulses have a much longer duration than $\tau_{e-p}$ and the heating and cooling take place simultaneously. For cavity formation, this means that the hollow particle remains hot for a longer period and consequently the cavity may collapse. For this reason, uptake of matter from the environment is mandatory to stabilize the cavities formed with nanosecond pulses. This has an important implication regarding efficiency, which is very low in the latter case $\left(17 \%\right.$ at best $\left.{ }^{34}\right)$ due to the strict conditions required to trap matter from the environment. ${ }^{34}$ In contrast, cavities formed via irradiation with femtosecond pulses and a fast quenching are kinetically stabilized, so we expect this method to have much higher efficiency. In addition, the single shot nature of the method will produce hollow nanoparticles with a shape dispersion much lower than that achieved with nanosecond pulse irradiation.

The underlying processes leading to the formation of these hollow nanoparticles are schematically summarized in Figure 1. Once the nanoparticle absorbs a femtosecond laser pulse, it melts and expands, forming an empty cavity inside. The molten particle then cools down following one of two possible pathways, depending on the cooling rate. During a slow cooling the 
particle evolves into its most thermodynamically stable form: a solid sphere. However, if a fast quenching takes place, the particle does not have sufficient time to return to its original shape in the short period that it remains hot. Thus, it is "frozen" in this metastable shape and the inner cavity is preserved.
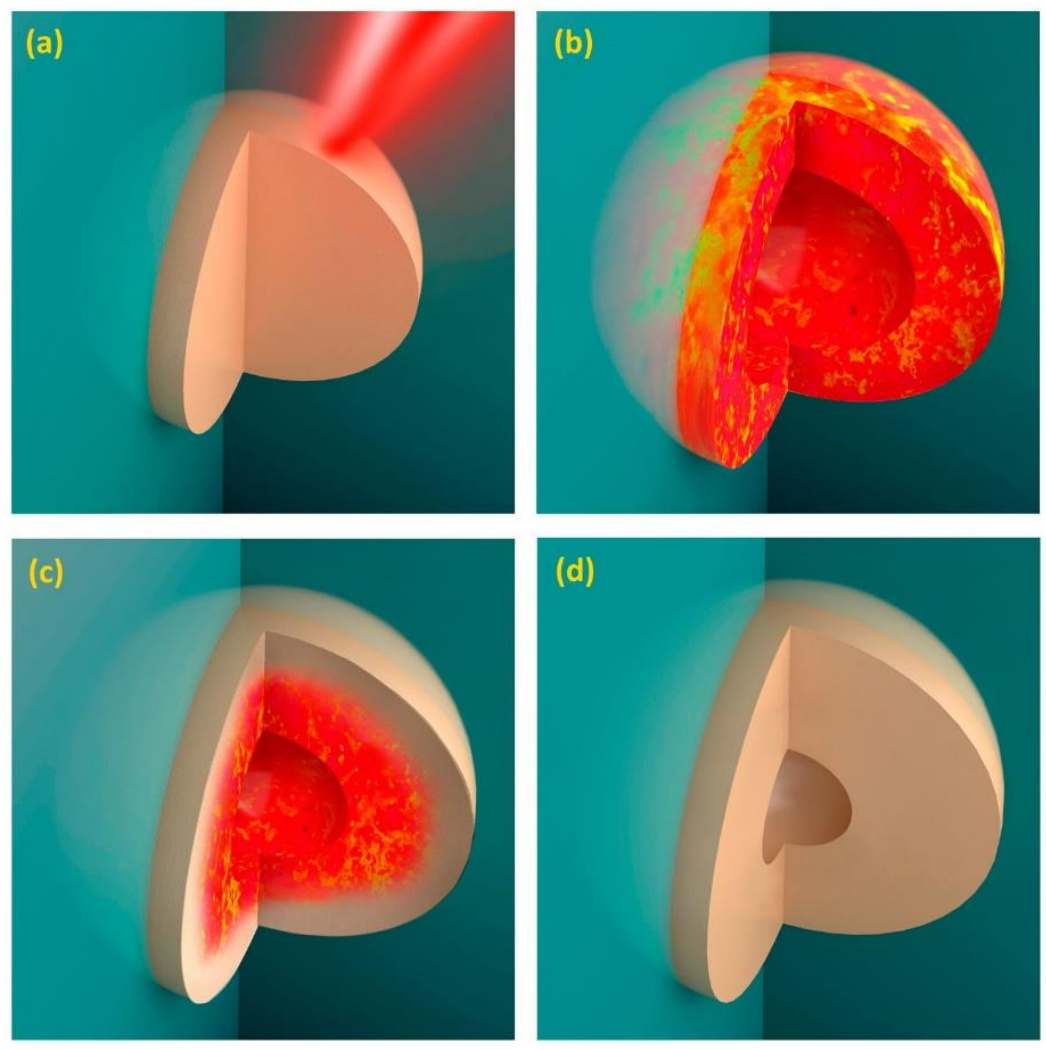

Figure 1. Schematic representation of the hollow nanosphere formation process under femtosecond laser irradiation. After (a) a solid nanoparticle is irradiated with a femtosecond laser pulse, (b) it melts and expands, forming a shell composed of molten material. Finally, (c) if the cooling process is fast enough, the particle "freezes" preserving the cavity formed at high temperature; eventually leading to (d) a stable hollow nanosphere. 


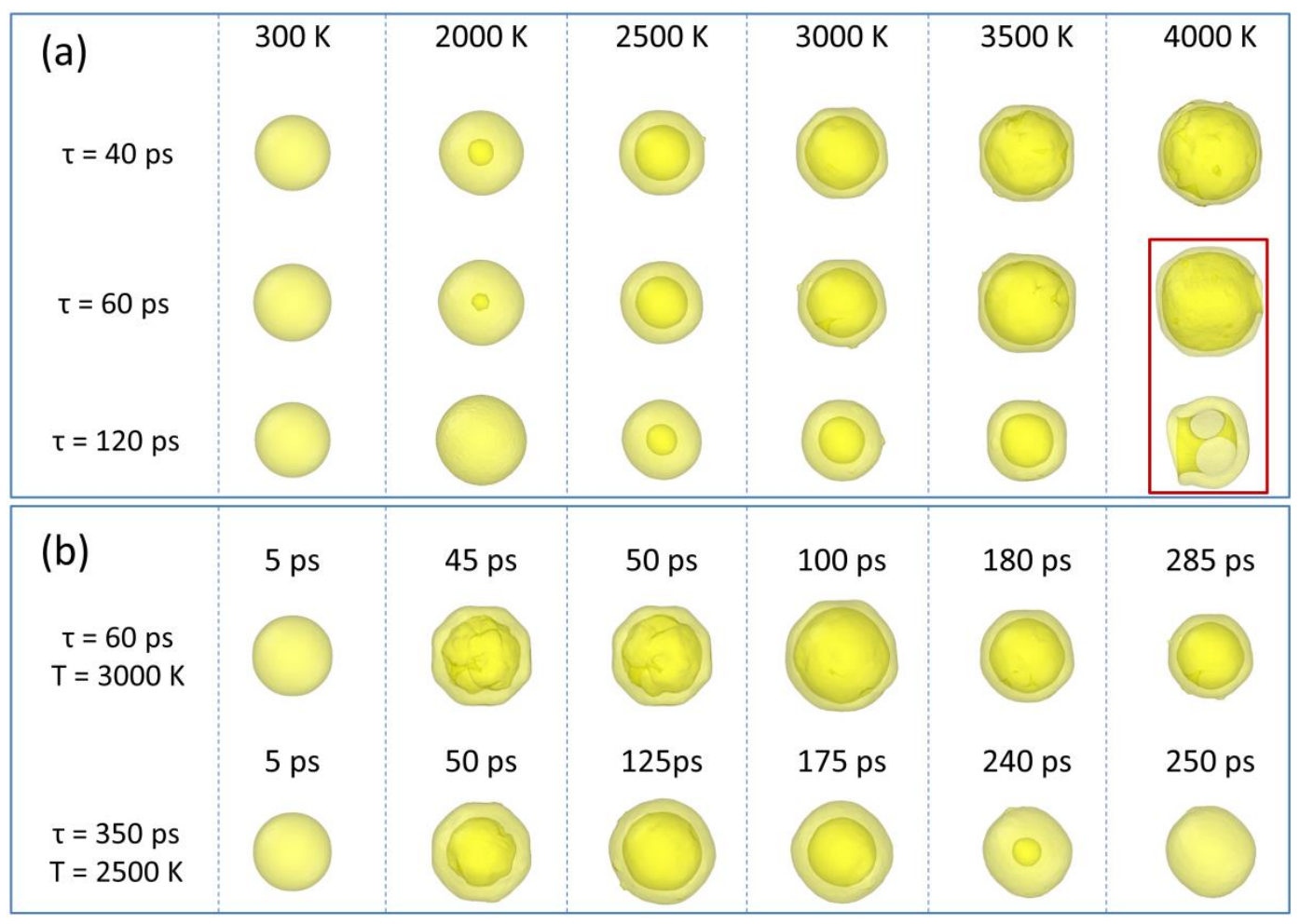

Figure 2. Summary of MD results. (a) Snapshots of the final configuration for $20 \mathrm{~nm}$ radius gold nanoparticles heated to the indicated temperature and cooled to $300 \mathrm{~K}$ using different values of the Langevin damping parameter, $\tau$. The images within the red rectangle indicate that, for this condition, some hundreds of atoms are ejected and, instead of a continuous shell, we obtain an irregular structure with several gaps in the wall. (b) Snapshots of intermediate evolution for $20 \mathrm{~nm}$ radius gold nanoparticles heated to the indicated temperature and cooled to $300 \mathrm{~K}$ using different values of $\tau$. The OVITO visualization software was used to generate the snapshots. ${ }^{38}$

Assuming that the initial particles have a small size dispersion, the process should efficiently produce near-identical hollow nanoparticles with a similar optical response. Therefore, a complex purification process to separate the desired hollow nanoparticles becomes unnecessary. The possibility of controlling the process of cavity formation leads to a complete control over the optical properties of these structures. This is a first step towards important applications, such as 
hydrogen trapping, storage, and release. For instance, one might speculate that by irradiating the particles in a medium with a high partial hydrogen pressure and/or replacing gold by palladium, one could promote hydrogen diffusion into the cavity while the lattice is hot. Clearly more work will be necessary to understand all the processes related to this problem, which is outside the scope of the present work. Finally, the possibility of concentrating the laser energy in a small region can be used, for instance, to activate a chemical reaction, weld nanoparticles together, or detach molecules previously attached to the nanoparticle. ${ }^{39}$

Selected snapshots of the MD simulations are depicted in Figure 2. In all cases (Fig. 2a), the initial nanospheres were heated up to the indicated maximum temperature, $T$, and cooled to room temperature with different values of the Langevin damping parameter, $\tau$. We can observe the formation of hollow nanoparticles with a spherical inner cavity of varying radius and, related to this, a shell with different thicknesses, depending on the irradiation conditions. Cavities are formed for $T=2500-3500 \mathrm{~K}$ and $\tau=40$, 60 , and $120 \mathrm{ps}$ whereas their formation is completely suppressed when heat dissipation to the solvent is too slow ( $\tau=350$ ps, not shown). More specifically (Fig. 2b), we observe that the inner cavities are always formed upon irradiation (i.e., heating) but they shrink during cooling, unless the quenching is fast enough. Higher temperatures lead to the ejection of some hundreds of atoms and yield an irregular structure that, instead of a continuous shell, has several gaps in the wall, unless a faster cooling is applied (e.g., a continuous and smooth shell is only possible at $4000 \mathrm{~K}$ if $\tau=40 \mathrm{ps}$ ).

Now, a warning about the results obtained is appropriate. We should be cautious with structures obtained for the highest temperatures. First, in this case we are close to (but probably below) the energy range where particles can fragment due to Coulomb explosion, ${ }^{40}$ which is not considered in our methodology. On the other hand, the reduced charge screening created by transiently excited hot electrons may weaken locally the interatomic bonding between $\mathrm{Au}$ ions. ${ }^{41,42}$ Hence, it may also be that the enhanced atom mobility produced by this phenomenon might yield larger cavities than those predicted by our model. Finally, bubble formation might occur for the higher temperatures, potentially affecting the cooling rate (i.e., cavity 
stabilization) but this should not be an issue for two reasons. First, Nguyen et al. have determined experimentally that bubbles appear around 300 ps after energy deposition. ${ }^{43}$ In the conditions of fast quenching (mandatory for cavity formation), this means that the particle should be already cold when the bubbles are formed. Second, a similar conclusion can be drawn from a simple energetic analysis. If we assume a nanoparticle with a radius of 20-40 $\mathrm{nm}$ and a latent heat of vaporization for water of $2.4 \times 10^{6} \mathrm{~J} / \mathrm{kg},{ }^{44}$ we obtain that the energy required to evaporate a 5 -nm-layer of water is $0.5-1.7 \mathrm{MeV}$. These values are in the same order of magnitude as the energy that we deposit on the particle to raise the lattice temperature, ensuring that the latter must have dropped considerably when the bubbles appear.

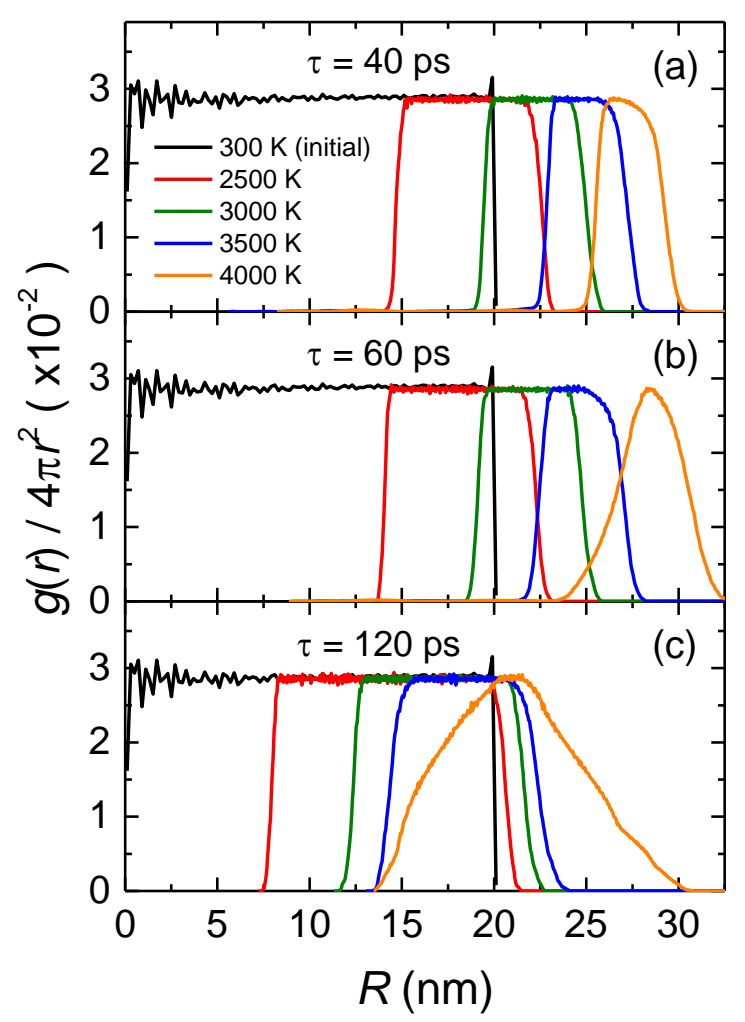

Figure 3. Radial distribution function for some selected temperatures and for different values of the Langevin damping parameter (a) $\tau=40 \mathrm{ps}$, (b) $\tau=60 \mathrm{ps}$ and (c) $\tau=120 \mathrm{ps.} \mathrm{The} \mathrm{initial} \mathrm{radial}$ distribution function at $300 \mathrm{~K}$ (black line) is included in all plots, as reference. 
From the radial distribution functions depicted in Figure 3, we can compare the morphology of the irradiated nanoparticles and determine the optimal conditions to form hollow nanoparticles. Only small morphological differences are noticed for the cases with $\tau=40$ and $60 \mathrm{ps,} \mathrm{except} \mathrm{for}$ the particles heated at $\sim 4000 \mathrm{~K}$, where only the fastest cooling rate enables the formation of hollow nanostructures with a well-defined shell. In general, a faster cooling rate promotes the formation of larger cavities whereas the opposite occurs for the shell thickness: highest $\tau$ values produce the thickest shells.

From these results, we can extract two significant observations. On one hand, the maximum temperature reached by the nanoparticle due to the laser excitation (i.e., directly related to the laser fluence) determines the formation of hollow nanostructures. Thus, no cavity is created at a temperature below $2000 \mathrm{~K}$, whereas for temperatures higher than $\sim 3500-4000 \mathrm{~K}$, atoms are ejected and irregular structures are obtained. On the other hand, the heat dissipation rate (represented in the model by the damping parameter) is the critical factor enabling the stabilization of hollow nanospheres formed after femtosecond laser excitation (i.e., further evolution is kinetically suppressed). Overall, these observations constitute the basis of a method for producing stable hollow nanoparticles by means of femtosecond irradiation of solid nanospheres. Our simulations indicate that the key experimental parameters are the laser fluence (energy deposited into the nanoparticle) and the cooling rate (the thermal properties of the matrix).

Interestingly, the aspect ratio of the hollow nanoparticle (i.e., the ratio of the cavity radius to that of the particle) can be controlled by a combination of laser fluence and cooling rate (the surfactant concentration for particles in an aqueous medium), as shown in Figure 4. High aspect ratios $(>0.6)$ are obtained for damping parameters of 40 and $60 \mathrm{ps}$ and temperatures above $2500 \mathrm{~K}$, whereas for $\tau=120 \mathrm{ps}$, relatively large $(>0.5)$ aspect ratios are also obtained. In this context, hollow 
nanoparticles with high aspect ratio are interesting for two reasons: (i) the optical response of the nanoparticle can be precisely tuned, as described next, and (ii) the cavities are large enough for storage of matter (e.g., hydrogen for energy applications).

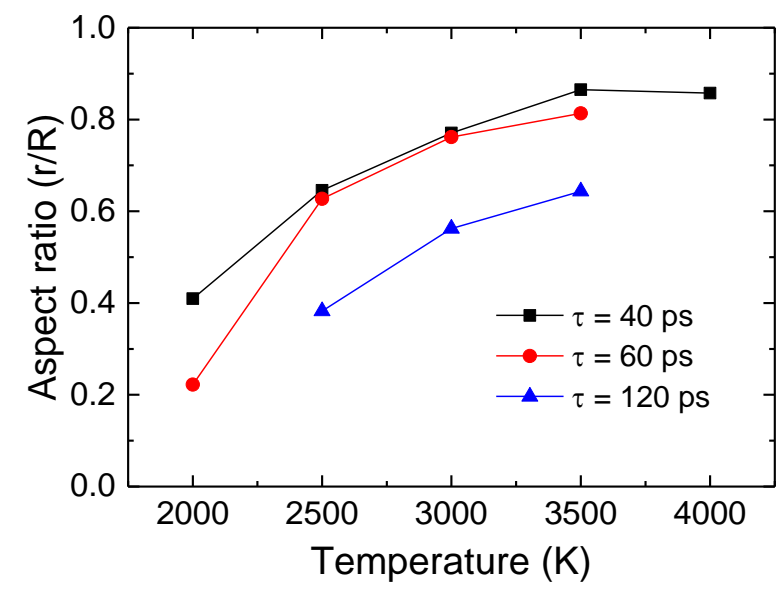

Figure 4. Aspect ratio of hollow nanospheres as a function of the maximum temperature reached after laser excitation, for different Langevin damping parameters.

In Figure 5 we show the optical response of hollow nanoparticles of different sizes as a function of their aspect ratio and wavelength. The LSPR position (maximum values in the color maps) only changes appreciably for aspect ratios above 0.6 . From that point onwards, the position of the LSPR depends strongly on the aspect ratio. This behavior can be better understood through the plasmon hybridization theory. ${ }^{45}$ In this approach, the LSPR of hollow nanoparticles can be viewed as the interaction between the plasmon modes of a sphere and a cavity. Their hybridization creates two new modes: antibonding and bonding, located at higher and lower energies, respectively. They correspond, respectively, to the antisymmetric and symmetric interactions between the plasmon modes of the sphere and the cavity ${ }^{45,46}$ The strength of the coupling is controlled by the shell thickness. Hence, the bonding plasmon mode (represented in Fig. 5) is redshifted as the shell gets thinner, and this effect becomes much more evident for aspect ratios above 0.6. According to our 
simulations, these conditions are reached for fast cooling (i.e., $\tau \leq 60 \mathrm{ps}$ ) and a maximum temperature above $2500 \mathrm{~K}$. On the other hand, a temperature of at least $3000 \mathrm{~K}$ is needed to attain a large aspect ratio with a somewhat slower cooling rate $(\tau=120 \mathrm{ps})$.
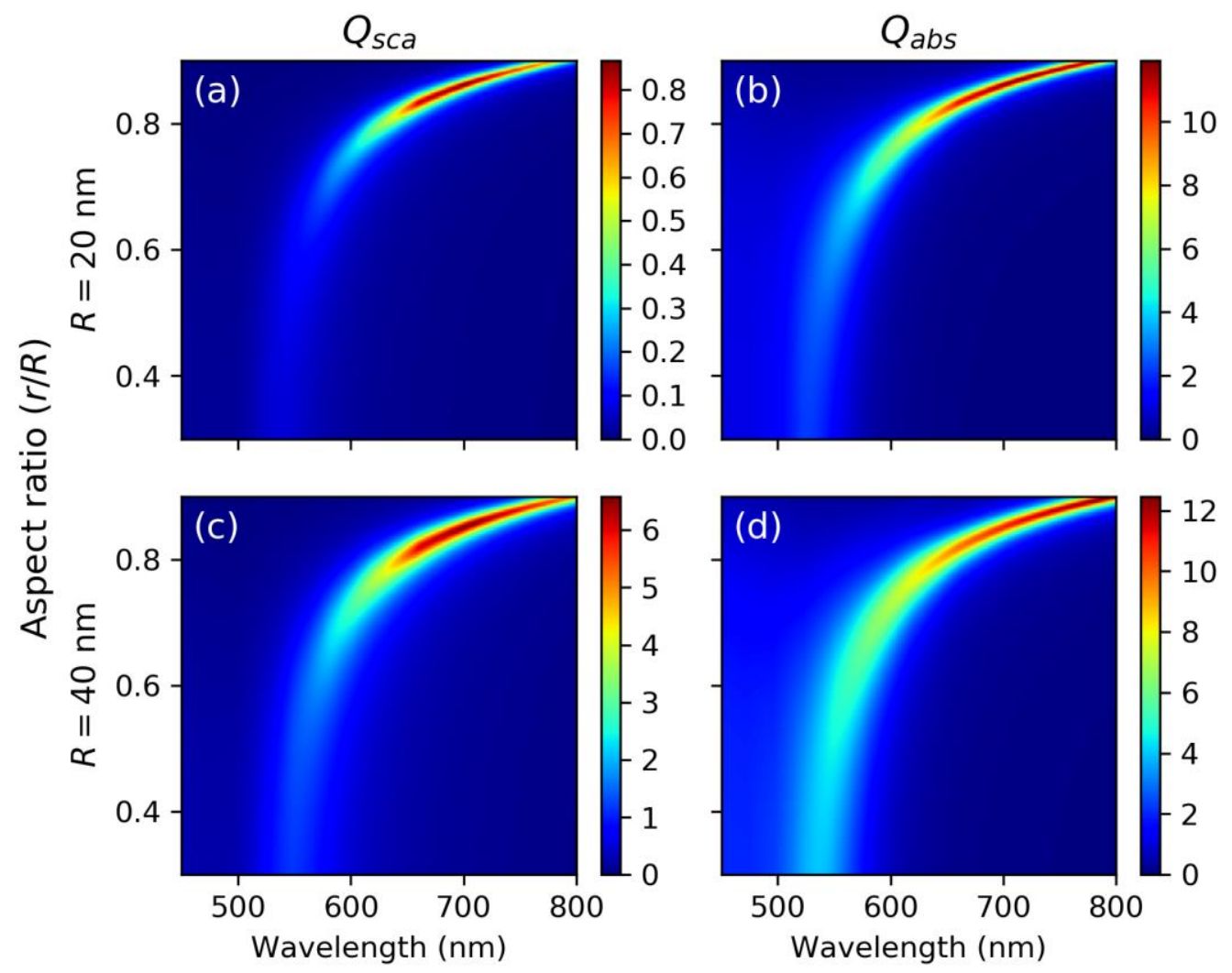

Figure 5. Scattering $\left(Q_{s c a}\right)$ and absorption $\left(Q_{a b s}\right)$ efficiencies of a hollow gold nanoparticle as a function of aspect ratio and light wavelength for nanoparticles of 20 and $40 \mathrm{~nm}$ radius. The upper row represents (a) the scattering and (b) the absorption efficiency for a particle with a radius of 20 $\mathrm{nm}$. The lower row represents (c) the scattering and (d) the absorption efficiency for a particle with a radius of $40 \mathrm{~nm}$.

It is important to connect the simulation parameters with those of the experiments for a successful application of the method to fabricate hollow nanoparticles. It is worth noting that, for nanoparticles in an aqueous environment, surfactants play a role as a thermal barrier affecting the 
cooling rate. In other words, the surfactant concentration is related to the exponential temperature decay, with very low concentrations leading to characteristic decay times of $\sim 60 \mathrm{ps},{ }^{47}$ whereas the critical micelle concentration has a decay time of $\sim 350 \mathrm{ps}$, as experimentally reported. ${ }^{43}$ However, these are approximate values and some optimization of the surfactant concentration may be required. Finally, it remains unclear if water is the best medium for the production of hollow nanoparticles. It has been reported that the cooling rate for gold nanoparticles embedded in poly(vinyl alcohol) is $40 \mathrm{ps},{ }^{48}$ which makes this matrix a very attractive candidate.

On the other hand, our results indicate that cavities are only formed if the maximum temperature reached by the nanoparticle after laser excitation is between 2500 and $3500 \mathrm{~K}$. Nevertheless, some experimental optimization will be needed to find the fluence required to produce hollow nanoparticles with the desired aspect ratio. The reason is that energy transfer efficiency from plasmons to the lattice can be as low as $75 \% .{ }^{4}$ For example, assuming a transfer efficiency in the range $70-90 \%$, the fluence range to increase the temperature of a 20 -nm-radius nanoparticle with $Q_{\text {abs }}=5$ from $300 \mathrm{~K}$ to $2500-3500 \mathrm{~K}$ is roughly between 30 and $60 \mathrm{~J} / \mathrm{m}^{2}$. Thus, the final dimensions of the resulting hollow nanoparticles are expected to depend on the fluence value. This is a strong point of the method because the laser fluence is an easy-to-change experimental parameter, which should enable straightforward tuning of the optical response of the hollow nanoparticle.

In summary, from classical molecular dynamics simulations, we have proposed a method to produce hollow nanoparticles using femtosecond laser irradiation. Our results indicate that cavity formation occurs at maximum temperatures in the range between 2500 and $3500 \mathrm{~K}$ (for 20-nmradius nanospheres, which translates into a laser fluence range between 30 and $60 \mathrm{~J} / \mathrm{m}^{2}$ ). Moreover, the cooling rate was found to be pivotal to stabilize the hollow nanostructures. Thus, a fast cooling 
rate $(\tau \leq 120 \mathrm{ps})$ is needed to avoid the collapse of the cavity during the relaxation process. In practice, the most reasonable value for the cooling rate of nanoparticles immersed in an aqueous solvent would be $\tau=60-120$ ps since some surfactant must be used to avoid nanoparticle aggregation in the colloid, which slows down the heat dissipation. Among different stabilizing agents, surfactants such as $\mathrm{CTAB}$ are probably the most attractive ones for practical implementation of this method, since they allow straightforward tuning of the cooling rate via variation of their concentration in solution. Solid matrices, like poly(vinyl alcohol), are also attractive due to their fast cooling rate $(40 \mathrm{ps}) .{ }^{48}$ The findings of this work can be put alongside the efforts of many research groups who are interested in developing potential applications of plasmonic nanoparticles in fields such as energy production and storage.

\section{COMPUTATIONAL METHODS}

Classical Molecular Dynamics (MD) simulations shown in this letter were performed with the program LAMMPS (Large-scale Atomic/Molecular Massively Parallel Simulator) from Sandia National Laboratories. ${ }^{49,50}$ Using MD, we simulated irradiation of colloidal nanoparticles (solid Au nanospheres) with femtosecond laser pulses. The system consisted of a fcc solid gold sphere with a radius of $20 \mathrm{~nm}$ (about $2 \times 10^{6}$ atoms) and at an initial temperature of $300 \mathrm{~K}$. Due to the fast energy deposition, we expect the particles to increase their temperature adiabatically during the pulse duration. Subsequently, heat dissipation leads to a rapid cooling, ${ }^{43}$ which can be roughly represented as an exponential decay. The process of energy deposition in the electronic system was not considered here because it occurs within a very short timescale compared to the electronphonon dynamics. Energy transfer to the lattice through electron-phonon coupling has been roughly considered as a linear increase of temperature up to a maximum value (between 2000 and 
$4000 \mathrm{~K}$ ) for $7 \mathrm{ps}$, which is the electron-phonon relaxation time for gold nanoparticles. ${ }^{51}$ This approach has been successfully used to describe the shape dynamics of gold nanorods after irradiation with femtosecond laser pulses, where the resulting aspect-ratios were in good agreement with those obtained experimentally. ${ }^{4}$ Moreover, using a rigorous approach that considers the contribution of d-band electrons via the density of states obtained from ab initio calculations, Lin and Zhigilei ${ }^{52}$ have shown that the increase of the lattice temperature occurs approximately at a similar time scale.

We should note that in this paper we were not concerned with the effects that occur during the transfer of energy from the electronic system to the crystal lattice. Due to the complex dependence of the electron-phonon coupling with the electronic temperature, ${ }^{52,53}$ it is unclear what laser fluence produces a specific lattice temperature. However, we just focused on the evolution of the lattice when it is heated to different temperatures without worrying about the fluences needed to reach them. Nevertheless, since fluence can easily be changed experimentally, this does not diminish the practical applications of this work.

Here the interaction between gold atoms was modelled by the EAM potential, ${ }^{54}$ which successfully reproduces several parameters, such as lattice properties, elastic constants, bulk modulus, Young's modulus, phonon frequencies, and the melting point. Heat dissipation from the particle to the solvent was represented with a Langevin thermostat. ${ }^{55}$ This thermostat models the interaction of the atoms with a background implicit solvent by means of three forces: (i) a conservative force, computed in this work via EAM potential, ${ }^{54}$ (ii) a frictional drag or viscous damping term $\left(F_{f}=-m \cdot v / \tau\right)$, where $m$ is the mass of Au atoms, $v$ their velocity, and $\tau$ a damping parameter, and (iii) a force due to solvent atoms at a temperature $T$ randomly bumping into the particle, which is proportional to $\sqrt{k_{B} \cdot T \cdot m /(\tau \cdot \Delta t)}$, where $k_{B}$ is the Boltzmann constant, and $\Delta t$ 
the simulation time step..$^{50}$ The damping parameter $\tau$ has time units and determines how rapidly the temperature is relaxed, which in this work should be considered as inversely related to the cooling rate of the matrix, a small relaxation time implying a high cooling rate and vice versa. Hence, thermal properties of dissimilar environments can be mimicked with different values of $\tau$. For example, an aqueous solvent can be depicted using $\tau=60 \mathrm{ps},{ }^{47}$ whereas $\tau=350 \mathrm{ps}$ has been experimentally found to represent a particle covered by surfactant molecules at the critical micelle concentration. ${ }^{43}$ In general, surfactant molecules act as a thermal barrier for heat dissipation; i.e., the higher the surfactant concentration, the higher the thermal barrier effect and, hence, larger $\tau$ values (slower cooling times). ${ }^{43}$ Solid matrices are also viable candidates, for instance, it has been reported that the cooling rate for gold nanoparticles embedded in poly(vinyl alcohol) matrix is 40 ps. ${ }^{48}$ The value of $120 \mathrm{ps}$ was added to provide an intermediate value between 60 and $350 \mathrm{ps}$ but it can represent experimentally, for example, nanoparticles in an aqueous solution with a CTAB concentration below the critical micelle concentration. The total simulation time varied between $350 \mathrm{ps}$ and $1 \mathrm{~ns}$, with a time step of $1 \mathrm{fs}$.

\section{ASSOCIATED CONTENT}

The Supporting Information is available free of charge at http://pubs.acs.org.

A video of an MD simulation that shows the evolution of a gold nanoparticle heated to $1800 \mathrm{~K}$ and with a cooling rate of $40 \mathrm{ps}$, where no cavity is formed (MP4).

A video of an MD simulation that shows the evolution of a gold nanoparticle heated to $2500 \mathrm{~K}$ and with a cooling rate of $350 \mathrm{ps}$, where the cavity is formed but it closes during the cooling (MP4). 
A video of an MD simulation that shows the evolution of a gold nanoparticle heated to $3500 \mathrm{~K}$

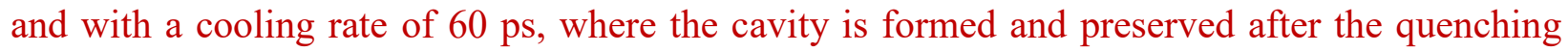
(MP4).

A video of an MD simulation that shows the evolution of a gold nanoparticle heated to $4000 \mathrm{~K}$

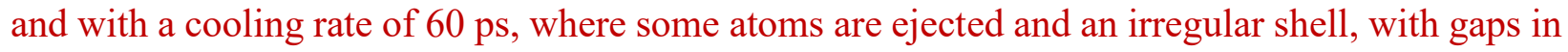
the wall, is formed (MP4).

\section{AUTHOR INFORMATION}

\section{Corresponding Authors}

Ovidio Peña-Rodríguez - Email: ovidio.pena@upm.es, ORCID: 0000-0002-7329-0550

Antonio Rivera - Email: antonio.rivera@upm.es, ORCID: 0000-0002-8484-5099

\section{Notes}

The authors declare no competing financial interests.

\section{ACKNOWLEDGMENT}

This work has been funded by the Spanish Ministry of Science, Innovation and Universities (MICIU) (grants RTI2018-095844-B-I00, PGC2018-096444-B-I00 and MAT2017-86659-R), the EUROfusion Consortium through project ENR-IFE19.CCFE-01 and the Madrid Regional Government (grants P2018/NMT-4389 and P2018/EMT-4437). A.P. thanks the support of FONDECYT under grants \#3190123 and \#11180557, as well as from Financiamiento Basal para Centros Científicos y Tecnológicos de Excelencia FB-0807. K.L. acknowledges the support of Russian Science Foundation (Project 19-19-00683). The authors acknowledge the computer resources and technical assistance provided by the Centro de Supercomputación y Visualización 
de Madrid (CeSViMa) and the supercomputing infrastructure of the NLHPC (ECM-02). This letter is based upon work from COST Action TUMIEE (CA17126). 


\section{REFERENCES}

(1) Zhang, Q.; Tan, Y. N.; Xie, J.; Lee, J. Y. Colloidal Synthesis of Plasmonic Metallic Nanoparticles. Plasmonics 2009, 4 (1), 9-22. https://doi.org/10.1007/s11468-008-9067-X.

(2) Coman, C.; Leopold, L. F.; Rugină, O. D.; Barbu-Tudoran, L.; Leopold, N.; Tofană, M.; Socaciu, C. Green Synthesis of Gold Nanoparticles by Allium Sativum Extract and Their Assessment as SERS Substrate. J. Nanopart. Res. 2013, 16 (1), 1-9. https://doi.org/10.1007/s11051-013-2158-4.

(3) Scarabelli, L. Recent Advances in the Rational Synthesis and Self-Assembly of Anisotropic Plasmonic Nanoparticles. Pure Appl. Chem. 2018, 90 (9), 1393-1407. https://doi.org/10.1515/pac-2018-0510.

(4) González-Rubio, G.; Díaz-Núñez, P.; Rivera, A.; Prada, A.; Tardajos, G.; GonzálezIzquierdo, J.; Bañares, L.; Llombart, P.; Macdowell, L. G.; Palafox, M. A.; Liz-Marzán, L. M.; Peña-Rodríguez, O.; Guerrero-Martínez, A. Femtosecond Laser Reshaping Yields Gold Nanorods with Ultranarrow Surface Plasmon Resonances. Science 2017, 358 (6363), 640-644. https://doi.org/10.1126/science.aan8478.

(5) Khlebtsov, N. G.; Dykman, L. A. Optical Properties and Biomedical Applications of Plasmonic Nanoparticles. J. Quant. Spectrosc. Radiat. Transf. 2010, 111 (1), 1-35. https://doi.org/10.1016/j.jqsrt.2009.07.012.

(6) Díaz, M.; Martínez, L.; Ruano, M. M.; P, D. L.; Román, E.; García-Hernandez, M.; Ballesteros, C.; Fermento, R.; Cebollada, A.; Armelles, G.; Huttel, Y. Morphological, Structural, and Magnetic Properties of Co Nanoparticles in a Silicon Oxide Matrix. $J$. Nanopart. Res. 2011, 13 (10), 5321-5333. https://doi.org/10.1007/s11051-011-0518-5.

(7) Rodriguez-Montelongo, S. A.; Gonzalez-Hernandez, J.; Macias, A. H.; Silva-Ramirez, A. S.; Castillo Martin del Campo, C. G.; Gutierrez-Hernandez, J. M.; Ruiz, F.; GonzalezOrtega, O. Synthesis, Characterization, and Toxicity of Hollow Gold Nanoshells. $J$. Nanopart. Res. 2018, 20 (11), 311. https://doi.org/10.1007/s11051-018-4420-2.

(8) Höller, R. P. M.; Dulle, M.; Thomä, S.; Mayer, M.; Steiner, A. M.; Förster, S.; Fery, A.; Kuttner, C.; Chanana, M. Protein-Assisted Assembly of Modular 3D Plasmonic 
Raspberry-like Core/Satellite Nanoclusters: Correlation of Structure and Optical Properties. ACS Nano 2016, 10 (6), 5740-5750. https://doi.org/10.1021/acsnano.5b07533.

(9) Herzog, J. B.; Knight, M. W.; Natelson, D. Thermoplasmonics: Quantifying Plasmonic Heating in Single Nanowires. Nano Lett. 2014, 14 (2), 499-503. https://doi.org/10.1021/nl403510u.

(10) Pirzadeh, Z.; Pakizeh, T.; Miljkovic, V.; Langhammer, C.; Dmitriev, A. PlasmonInterband Coupling in Nickel Nanoantennas. ACS Photonics 2014, 1 (3), 158-162. https://doi.org/10.1021/ph4000339.

(11) Walters, C. M.; Pao, C.; Gagnon, B. P.; Zamecnik, C. R.; Walker, G. C. Bright SurfaceEnhanced Raman Scattering with Fluorescence Quenching from Silica Encapsulated JAggregate Coated Gold Nanoparticles. Adv. Mater. 2018, 30 (5), 1705381. https://doi.org/10.1002/adma.201705381.

(12) Ma, W.; Mao, J.; Hao, C.; Xu, L.; Xu, C.; Kuang, H. Chiral Semiconductor Nanorod Heterostructures with High Photocatalysis Activity. Appl. Catal. B 2019, 245, 691-697. https://doi.org/10.1016/j.apcatb.2019.01.038.

(13) Steinlechner, C.; Junge, H. Renewable Methane Generation from Carbon Dioxide and Sunlight. Angew. Chem. Int. Ed. 2018, 57 (1), 44-45. https://doi.org/10.1002/anie.201709032.

(14) Lee, J.-H.; Cho, H.-Y.; Choi, H. K.; Lee, J.-Y.; Choi, J.-W. Application of Gold Nanoparticle to Plasmonic Biosensors. Int. J. Mol. Sci. 2018, 19 (7), 2021. https://doi.org/10.3390/ijms 19072021.

(15) Argyropoulos, C.; Monticone, F.; D’Aguanno, G.; Alù, A. Plasmonic Nanoparticles and Metasurfaces to Realize Fano Spectra at Ultraviolet Wavelengths. Appl. Phys. Lett. 2013, 103 (14), 143113. https://doi.org/10.1063/1.4823575.

(16) Jiang, J.; Li, H.; Zhang, L. New Insight into Daylight Photocatalysis of AgBr@Ag: Synergistic Effect between Semiconductor Photocatalysis and Plasmonic Photocatalysis. Chem. - Eur. J. 2012, 18 (20), 6360-6369. https://doi.org/10.1002/chem.201102606.

(17) Li, S.; Zhang, J.; Kibria, M. G.; Mi, Z.; Chaker, M.; Ma, D.; Nechache, R.; Rosei, F. Remarkably Enhanced Photocatalytic Activity of Laser Ablated Au Nanoparticle 
Decorated $\mathrm{BiFeO}_{3}$ Nanowires under Visible-Light. Chem. Commun. 2013, 49 (52), 58565858. https://doi.org/10.1039/C3CC40363G.

(18) Priebe, J. B.; Karnahl, M.; Junge, H.; Beller, M.; Hollmann, D.; Brückner, A. Water Reduction with Visible Light: Synergy between Optical Transitions and Electron Transfer in $\mathrm{Au}-\mathrm{TiO}_{2}$ Catalysts Visualized by in Situ EPR Spectroscopy. Angew. Chem. Int. Ed. 2013, 52 (43), 11420-11424. https://doi.org/10.1002/anie.201306504.

(19) Yu, C.; Li, G.; Kumar, S.; Kawasaki, H.; Jin, R. Stable $\mathrm{Au}_{25}(\mathrm{SR})_{18} / \mathrm{TiO}_{2} \mathrm{Composite}$ Nanostructure with Enhanced Visible Light Photocatalytic Activity. J. Phys. Chem. Lett. 2013, 4 (17), 2847-2852. https://doi.org/10.1021/jz401447w.

(20) Miljevic, M.; Geiseler, B.; Bergfeldt, T.; Bockstaller, P.; Fruk, L. Enhanced Photocatalytic Activity of $\mathrm{Au} / \mathrm{TiO}_{2}$ Nanocomposite Prepared Using Bifunctional Bridging Linker. Adv. Funct. Mater. 2014, 24 (7), 907-915. https://doi.org/10.1002/adfm.201301484.

(21) Yayama, T.; Ishimoto, T.; Koyama, M. Theoretical Investigation of Hydrogen Absorption Properties of Rhodium-Silver Alloys. J. Alloys Compd. 2016, 662, 404-408. https://doi.org/10.1016/j.jallcom.2015.12.008.

(22) Maier, S. A. Plasmonics: Fundamentals and Applications, 1st ed.; Springer, 2007.

(23) Gong, C.; Kaplan, A.; Benson, Z. A.; Baker, D. R.; McClure, J. P.; Rocha, A. R.; Leite, M. S. Band Structure Engineering by Alloying for Photonics. Adv. Opt. Mater. 2018, 6 (17), 1800218. https://doi.org/10.1002/adom.201800218.

(24) Scotognella, F.; Della Valle, G.; Srimath Kandada, A. R.; Dorfs, D.; Zavelani-Rossi, M.; Conforti, M.; Miszta, K.; Comin, A.; Korobchevskaya, K.; Lanzani, G.; Manna, L.; Tassone, F. Plasmon Dynamics in Colloidal $\mathrm{Cu}_{2-\mathrm{X}} \mathrm{Se}$ Nanocrystals. Nano Lett. 2011, 11 (11), 4711-4717. https://doi.org/10.1021/n1202390s.

(25) Luther, J. M.; Jain, P. K.; Ewers, T.; Alivisatos, A. P. Localized Surface Plasmon Resonances Arising from Free Carriers in Doped Quantum Dots. Nat. Mater. 2011, 10 (5), 361-366. https://doi.org/10.1038/nmat3004.

(26) Li, W.; Zamani, R.; Rivera Gil, P.; Pelaz, B.; Ibáñez, M.; Cadavid, D.; Shavel, A.; Alvarez-Puebla, R. A.; Parak, W. J.; Arbiol, J.; Cabot, A. CuTe Nanocrystals: Shape and 
Size Control, Plasmonic Properties, and Use as SERS Probes and Photothermal Agents. $J$. Am. Chem. Soc. 2013, 135 (19), 7098-7101. https://doi.org/10.1021/ja401428e.

(27) Liu, X.; Wang, X.; Zhou, B.; Law, W.-C.; Cartwright, A. N.; Swihart, M. T. SizeControlled Synthesis of $\mathrm{Cu}_{2-\mathrm{x}} \mathrm{E}(\mathrm{E}=\mathrm{S}, \mathrm{Se})$ Nanocrystals with Strong Tunable nearInfrared Localized Surface Plasmon Resonance and High Conductivity in Thin Films. Adv. Funct. Mater. 2013, 23 (10), 1256-1264. https://doi.org/10.1002/adfm.201202061.

(28) Kanehara, M.; Koike, H.; Yoshinaga, T.; Teranishi, T. Indium Tin Oxide Nanoparticles with Compositionally Tunable Surface Plasmon Resonance Frequencies in the Near-IR Region. J. Am. Chem. Soc. 2009, 131 (49), 17736-17737. https://doi.org/10.1021/ja9064415.

(29) Manthiram, K.; Beberwyck, B. J.; Alivisatos, A. P. Enhanced Electrochemical Methanation of Carbon Dioxide with a Dispersible Nanoscale Copper Catalyst. J. Am. Chem. Soc. 2014, 136 (38), 13319-13325. https://doi.org/10.1021/ja5065284.

(30) González-Rubio, G.; González-Izquierdo, J.; Bañares, L.; Tardajos, G.; Rivera, A.; Altantzis, T.; Bals, S.; Peña-Rodríguez, O.; Guerrero-Martínez, A.; Liz-Marzan, L. M. Femtosecond Laser-Controlled Tip-to-Tip Assembly and Welding of Gold Nanorods. Nano Lett. 2015, 15 (12), 8282-8288. https://doi.org/10.1021/acs.nanolett.5b03844.

(31) Peña-Rodríguez, O.; Prada, A.; Olivares, J.; Oliver, A.; Rodríguez-Fernández, L.; SilvaPereyra, H. G.; Bringa, E.; Perlado, J. M.; Rivera, A. Understanding the Ion-Induced Elongation of Silver Nanoparticles Embedded in Silica. Sci. Rep. 2017, 7 (1), 922. https://doi.org/10.1038/s41598-017-01145-0.

(32) Grossi, J.; Kohanoff, J.; Todorov, T. N.; Artacho, E.; Bringa, E. M. Electronic Heat Transport versus Atomic Heating in Irradiated Short Metallic Nanowires. Phys. Rev. B 2019, 100 (15), 155434. https://doi.org/10.1103/PhysRevB.100.155434.

(33) Díaz-Nuñez, P.; González-Rubio, G.; Prada, A.; González Izquierdo, J.; Rivera, A.; Bañares, L.; Guerrero-Martínez, A.; Peña-Rodríguez, O. Using Femtosecond Laser Irradiation to Grow the Belly of Gold Nanorods. J. Phys. Chem. C 2018, 122 (34), 19816-19822. https://doi.org/10.1021/acs.jpcc.8b06375. 
(34) González-Rubio, G.; Milagres de Oliveira, T.; Albrecht, W.; Díaz-Núñez, P.; CastroPalacio, J. C.; Prada, A.; Gonzalez, R. I.; Scarabelli, L.; Banares, L.; Rivera, A.; LizMarzán, L. M.; Peña-Rodríguez, O.; Bals, S.; Guerrero-Martínez, A. Formation of Hollow Gold Nanocrystals by Nanosecond Laser Irradiation. J. Phys. Chem. Lett. 2020, 11 (3), 670-677. https://doi.org/10.1021/acs.jpclett.9b03574.

(35) Hou, B.; Shen, L.; Shi, H.; Kapadia, R.; Cronin, S. B. Hot Electron-Driven Photocatalytic Water Splitting. Phys. Chem. Chem. Phys. 2017, 19 (4), 2877-2881. https://doi.org/10.1039/C6CP07542H.

(36) Naldoni, A.; Guler, U.; Wang, Z.; Marelli, M.; Malara, F.; Meng, X.; Besteiro, L. V.; Govorov, A. O.; Kildishev, A. V.; Boltasseva, A.; Shalaev, V. M. Broadband HotElectron Collection for Solar Water Splitting with Plasmonic Titanium Nitride. Adv. Opt. Mater. 2017, 5 (15), 1601031. https://doi.org/10.1002/adom.201601031.

(37) Metwally, K.; Mensah, S.; Baffou, G. Fluence Threshold for Photothermal Bubble Generation Using Plasmonic Nanoparticles. J. Phys. Chem. C 2015, 119 (51), 2858628596. https://doi.org/10.1021/acs.jpcc.5b09903.

(38) Stukowski, A. Visualization and Analysis of Atomistic Simulation Data with OVITO-the Open Visualization Tool. Modelling Simul. Mater. Sci. Eng. 2009, 18 (1), 015012. https://doi.org/10.1088/0965-0393/18/1/015012.

(39) Kim, M.; Lin, M.; Son, J.; Xu, H.; Nam, J.-M. Hot-electron-mediated Photochemical Reactions: Principles, Recent Advances, and Challenges. Adv. Opt. Mater. 2017, 5 (15), 1700004. https://doi.org/10.1002/adom.201700004.

(40) Werner, D.; Furube, A.; Okamoto, T.; Hashimoto, S. Femtosecond Laser-Induced Size Reduction of Aqueous Gold Nanoparticles: In Situ and Pump-Probe Spectroscopy Investigations Revealing Coulomb Explosion. J. Phys. Chem. C 2011, 115 (17), 85038512. https://doi.org/10.1021/jp112262u.

(41) Daraszewicz, S. L.; Giret, Y.; Naruse, N.; Murooka, Y.; Yang, J.; Duffy, D. M.; Shluger, A. L.; Tanimura, K. Structural Dynamics of Laser-Irradiated Gold Nanofilms. Phys. Rev. B 2013, 88 (18), 184101. https://doi.org/10.1103/PhysRevB.88.184101. 
(42) Ihm, Y.; Cho, D. H.; Sung, D.; Nam, D.; Jung, C.; Sato, T.; Kim, S.; Park, J.; Kim, S.; Gallagher-Jones, M.; Kim, Y.; Xu, R.; Owada, S.; Shim, J. H.; Tono, K.; Yabashi, M.; Ishikawa, T.; Miao, J.; Noh, D. Y.; Song, C. Direct Observation of Picosecond Melting and Disintegration of Metallic Nanoparticles. Nat. Commun. 2019, 10 (1), 1-6. https://doi.org/10.1038/s41467-019-10328-4.

(43) Nguyen, S. C.; Zhang, Q.; Manthiram, K.; Ye, X.; Lomont, J. P.; Harris, C. B.; Weller, H.; Alivisatos, A. P. Study of Heat Transfer Dynamics from Gold Nanorods to the Environment via Time-Resolved Infrared Spectroscopy. ACS Nano 2016, 10 (2), 21442151. https://doi.org/10.1021/acsnano.5b06623.

(44) Henderson-Sellers, B. A New Formula for Latent Heat of Vaporization of Water as a Function of Temperature. Q. J. R. Meteorol. Soc. 1984, 110 (466), 1186-1190. https://doi.org/10.1002/qj.49711046626.

(45) Prodan, E.; Radloff, C.; Halas, N. J.; Nordlander, P. A Hybridization Model for the Plasmon Response of Complex Nanostructures. Science 2003, 302 (5644), 419-422. https://doi.org/10.1126/science.1089171.

(46) Peña, O.; Pal, U.; Rodríguez-Fernández, L.; Crespo-Sosa, A. Linear Optical Response of Metallic Nanoshells in Different Dielectric Media. J. Opt. Soc. Am. B 2008, 25 (8), 13711379. https://doi.org/10.1364/JOSAB.25.001371.

(47) Ekici, O.; Harrison, R. K.; Durr, N. J.; Eversole, D. S.; Lee, M.; Ben-Yakar, A. Thermal Analysis of Gold Nanorods Heated with Femtosecond Laser Pulses. J. Phys. D: Appl. Phys. 2008, 41 (18), 185501. https://doi.org/10.1088/0022-3727/41/18/185501.

(48) Wang, L.; Takeda, S.; Liu, C.; Tamai, N. Coherent Acoustic Phonon Dynamics of Gold Nanorods and Nanospheres in a Poly(Vinyl Alcohol) Matrix and Their Temperature Dependence by Transient Absorption Spectroscopy. J. Phys. Chem. C 2014, 118 (3), 1674-1681. https://doi.org/10.1021/jp4100679.

(49) Plimpton, S. Fast Parallel Algorithms for Short-Range Molecular Dynamics. J. Comput. Phys. 1995, 117 (1), 1-19. https://doi.org/10.1006/jcph.1995.1039.

(50) LAMMPS Molecular Dynamics Simulator https://lammps.sandia.gov/ (accessed Jul 5, 2019). 
(51) Zhang, J. Z. Ultrafast Studies of Electron Dynamics in Semiconductor and Metal Colloidal Nanoparticles: Effects of Size and Surface. Acc. Chem. Res. 1997, 30 (10), 423-429. https://doi.org/10.1021/ar960178j.

(52) Lin, Z.; Zhigilei, L. V. Thermal Excitation of d Band Electrons in Au: Implications for Laser-Induced Phase Transformations. In High-Power Laser Ablation VI; International Society for Optics and Photonics, 2006; Vol. 6261, p 62610U. https://doi.org/10.1117/12.674636.

(53) Mongin, D.; Maioli, P.; Burgin, J.; Langot, P.; Cottancin, E.; D’Addato, S.; Canut, B.; Treguer, M.; Crut, A.; Vallée, F.; Fatti, N. D. Ultrafast Electron-Lattice Thermalization in Copper and Other Noble Metal Nanoparticles. J. Phys.: Condens. Matter 2019, 31 (8), 084001. https://doi.org/10.1088/1361-648X/aaf7eb.

(54) Lee, B.-J.; Shim, J.-H.; Baskes, M. I. Semiempirical Atomic Potentials for the Fcc Metals $\mathrm{Cu}, \mathrm{Ag}, \mathrm{Au}, \mathrm{Ni}, \mathrm{Pd}, \mathrm{Pt}, \mathrm{Al}$, and $\mathrm{Pb}$ Based on First and Second Nearest-Neighbor Modified Embedded Atom Method. Phys. Rev. B 2003, 68 (14), 144112. https://doi.org/10.1103/PhysRevB.68.144112.

(55) Schneider, T.; Stoll, E. Molecular-Dynamics Study of a Three-Dimensional OneComponent Model for Distortive Phase Transitions. Phys. Rev. B 1978, 17 (3), 1302 1322. https://doi.org/10.1103/PhysRevB.17.1302. 